The Astrophysical Journal, 649:389-398, 2006 September 20

(C) 2006. The American Astronomical Society. All rights reserved. Printed in U.S.A.

\title{
MASSES OF ASTROMETRICALLY DISCOVERED AND IMAGED BINARIES: G78-28AB AND GJ 231.1BC
}

\author{
Steven H. Pravdo \\ Jet Propulsion Laboratory, California Institute of Technology, 306-431, 4800 Oak Grove Drive, Pasadena, CA 91109; spravdo@jpl.nasa.gov \\ Stuart B. Shaklan \\ Jet Propulsion Laboratory, California Institute of Technology, 301-486, 4800 Oak Grove Drive, Pasadena, CA 91109; \\ shaklan@huey.jpl.nasa.gov \\ SLOANE J. WiKTOROWicz \\ Division of Geological and Planetary Sciences, California Institute of Technology, Pasadena, CA 91125; sloane@gps.caltech.edu \\ SHRI KULKARNI \\ Division of Physics, Mathematics, and Astronomy, California Institute of Technology, Pasadena, CA 91125; srk@astro.caltech.edu \\ James P. Lloyd and Frantz Martinache \\ Department of Astronomy, Cornell University, Ithaca, NY 14853; jpl@astro.cornell.edu, frantz@astro.cornell.edu
}

Peter G. Tuthill

School of Physics, University of Sydney, NSW 2006, Australia; gekko@physics.usyd.edu.au

AND

Michael J. IRELAND

Division of Geological and Planetary Sciences, California Institute of Technology,

Pasadena, CA 91125; mireland@gps.caltech.edu

Received 2006 April 12; accepted 2006 May 11

\begin{abstract}
The Stellar Planet Survey (STEPS) is an ongoing astrometric search for giant planets and brown dwarfs around a sample of $\sim 30 \mathrm{M}$ dwarfs. We have discovered several low-mass companions by measuring the motion of our target stars relative to their reference frames. The highest mass discovery thus far is G78-28B, a companion to the M dwarf G78-28A. The orbital period is $4.18 \pm 0.03 \mathrm{yr}$, the system mass is $0.565 \pm 0.055 M_{\odot}$, and the semimajor axis is $2.19 \pm 0.10 \mathrm{AU}$. Imaging observations with the Keck laser guide star adaptive optics (LGSAO) and the Palomar AO instruments resolved the system and also yielded $J H K$-band delta magnitudes. We use the orbital solution, light ratios, and mass-luminosity relationships to derive component masses of $M_{A}=0.370 \pm 0.034 M_{\odot}$ and $M_{B}=$ $0.195 \pm 0.021 M_{\odot}$. G78-28B is of type M4 V based on its colors and mass. We also discovered GJ 231.1C, a companion to GJ 231.1B, with STEPS and imaged the companion with LGSAO and Palomar AO, but the orbital period is longer than our observing baseline; thus, the system parameters are less constrained. In GJ 231.1BC the masses are $M_{B}=0.25 \pm 0.06 M_{\odot}$ and $M_{C}=0.12 \pm 0.02 M_{\odot}$. The inferred spectral type of GJ 231.1C is M5 V. We demonstrate the results of the current state of mass estimation techniques with our data.
\end{abstract}

Subject headings: planetary systems — stars: individual (G78-28A)

\section{INTRODUCTION}

The Stellar Planet Survey (STEPS) is an astrometric search for low-mass companions to M dwarfs. Astrometry provides a direct measurement of stellar mass because the full three-dimensional orbit is observed. Measurements of mass test and assist the development of the models based on parameters such as age and metallicity. Determining an accurate mass thus deepens our understanding of the fundamental physics of stars and substellar objects. Another direct benefit is to advance our knowledge of the mass-luminosity relationships (MLRs). At present there are no extant observational MLRs for brown dwarfs (BDs), and the MLR for stars at the bottom of the main sequence is based on only 10 objects (Henry et al. 1999). We have already made several mass measurements of companions to M dwarfs with STEPS (Pravdo et al. 2004, 2005a, 2005b). In each case the combination of astrometry and imaging resulted in conclusions about the masses of the components that could not have been reached by either technique alone.
Here we report on two more of the $\sim 30$ targets that at the beginning of the STEPS program were considered "single" stars. We astrometrically discovered companions around both G78-28 and GJ 231.1B, and we later confirmed the existence of the companions with laser guide star adaptive optics (LGSAO) and Palomar adaptive optics (AO) imaging observations. We present these results and discuss how they contribute to MLRs in particular and stellar knowledge in general.

\section{OBSERVATIONS AND RESULTS}

\subsection{Astrometry}

\subsection{1. $G 78-28 A B$}

G78-28 (=G95-22, LTT 17492, GJ 3213) is an M3 dwarf with the properties listed in Table 1. The parallax has not been determined trigonometrically and is estimated from the spectral type and colors as $66 \pm 13$ mas (Gliese \& Jahreiss 1991) and 59 mas (with large error; Reid et al. 1995). We observed it from 
TABLE 1

Previously Known Properties

\begin{tabular}{|c|c|c|}
\hline Property & G78-28 & GJ 231.1B \\
\hline R.A. $(\mathrm{J} 2000.0)^{\mathrm{a}}$ & $03 \quad 17 \quad 12.24$ & 061710.65 \\
\hline Decl. $(\mathrm{J} 2000.0)^{\mathrm{a}}$ & +452222.0 & +050600.4 \\
\hline$V^{\mathrm{b}} \ldots \ldots \ldots \ldots \ldots \ldots \ldots$ & 12.39 & 13.27 \\
\hline$J^{\mathrm{c}}$. & $8.422 \pm 0.023$ & $9.088 \pm 0.015$ \\
\hline$H^{\mathrm{c}} \ldots$ & $7.865 \pm 0.019$ & $8.559 \pm 0.039$ \\
\hline$K^{\mathrm{c}}$. & $7.593 \pm 0.013$ & $8.267 \pm 0.011$ \\
\hline Type...... & M3 & M3.5 \\
\hline 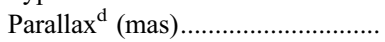 & $66 \pm 13$ & $50 \pm 10$ \\
\hline 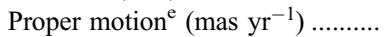 & $264 \pm 7$ & $287 \pm 22$ \\
\hline Position angle $^{\mathrm{e}}$ (deg)...................... & $253 \pm 3$ & $304 \pm 4$ \\
\hline
\end{tabular}

NoтE.- Units of right ascension are hours, minutes, and seconds, and units of declination are degrees, arcminutes, and arcseconds.

a Salim \& Gould (2003); Lepine et al. (2005).

b Weis (1988); Weistrop (1981).

c 2 MASS.

d Gliese \& Jahreiss (1991); VizieR Online Data Catalog, 174 (W. F. van Altena, J. T. Lee, \& E. D. Hoffleit, 1995); Reid et al. (1995).

e Luyten (1979); VizieR Online Data Catalog, 256 (CMC, 1999); Salim \& Gould (2003); Lepine et al. (2005).

1997 December through 2005 January with the STEPS instrument (astrometric bandpass 550-750 nm) mounted at the Cassegrain focus of the Palomar 200 inch $(5 \mathrm{~m})$ telescope. The first observation was 1997 December 21.3 = JD 2,450,803.8. Pravdo et al. (2004, 2005b) give more detailed descriptions of the instrument and data analysis.

Table 2 shows the results of our measurements of parallax and proper motion. Our parallax is measured relative to the in-frame reference and should be corrected for the reference frame's finite distance by the addition of $\sim 2 \pm 1$ mas for average fields at this Galactic latitude and apparent magnitude. ${ }^{1}$ The result is $\pi=$ $54.4 \pm 1.0$ mas. Our value is on the lower boundaries of the prior measurements, consistent with the fact that the primary star is now $0.2-0.3$ mag fainter in $V J H K$ than when it was believed to be the origin of all the light. Our proper motion values are consistent with prior results in which the error bars on the prior results are estimated from the variation among past observers (Luyten 1979; Salim \& Gould 2003). ${ }^{2}$ Proper motions of reference stars

1 VizieR Online Data Catalog, 174 (W. F. van Altena, J. T. Lee, \& E. D. Hoffleit, 1995).

2 VizieR Online Data Catalog, 256 (CMC, 1999).

TABLE 2

Derived Stellar and System Parameters

\begin{tabular}{|c|c|c|}
\hline Parameter & G78-28AB & GJ 231.1BC \\
\hline Relative parallax (mas). & $52.4 \pm 0.1$ & $53.2 \pm 0.2$ \\
\hline Absolute parallax (mas). & $54.4 \pm 1.0$ & $55.2 \pm 1.0$ \\
\hline Proper motion $\left(\operatorname{mas} \mathrm{yr}^{-1}\right)$.. & $269.3 \pm 0.3$ & $270.2 \pm 3.6$ \\
\hline Position angle $(\mathrm{deg}) .$. & $253.3 \pm 0.2$ & $309.8 \pm 3.6$ \\
\hline Period $(\mathrm{yr}) \ldots \ldots \ldots \ldots \ldots . . . . . .$. & $4.18 \pm 0.03$ & $>25.7$ \\
\hline Total mass $\left(M_{\odot}\right) \ldots \ldots \ldots \ldots \ldots$ & $0.53 \pm 0.09$ & $0.37 \pm 0.07$ \\
\hline Semimajor axis $(\mathrm{AU}) \ldots . .$. & $2.19 \pm 0.10$ & $>6.4$ \\
\hline Eccentricity, e & $0.281 \pm 0.030$ & $\ldots$ \\
\hline Inclination $(\mathrm{deg}) \ldots$ & $78 \pm 1$ & $90 \pm 3$ \\
\hline Longitude of ascending node ${ }^{\mathrm{a}}(\mathrm{deg}) \ldots \ldots .$. & $4.5 \pm 0.5$ & $\ldots$ \\
\hline 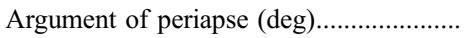 & $254.5 \pm 1.5$ & $\ldots$ \\
\hline Epoch ..................... & $2002.015 \pm 0.035$ & $\ldots$ \\
\hline Primary mass, $M_{\text {pri }}\left(M_{\odot}\right) \ldots \ldots \ldots \ldots \ldots \ldots \ldots \ldots \ldots \ldots \ldots \ldots \ldots \ldots$ & $0.370 \pm 0.034$ & $0.25 \pm 0.06$ \\
\hline Secondary mass, $M_{\mathrm{sec}}\left(M_{\odot}\right) \ldots \ldots \ldots \ldots \ldots \ldots$ & $0.195 \pm 0.021$ & $0.12 \pm 0.02$ \\
\hline
\end{tabular}

${ }^{\text {a }}$ Or $+180^{\circ}$ because of into or out of plane ambiguity.

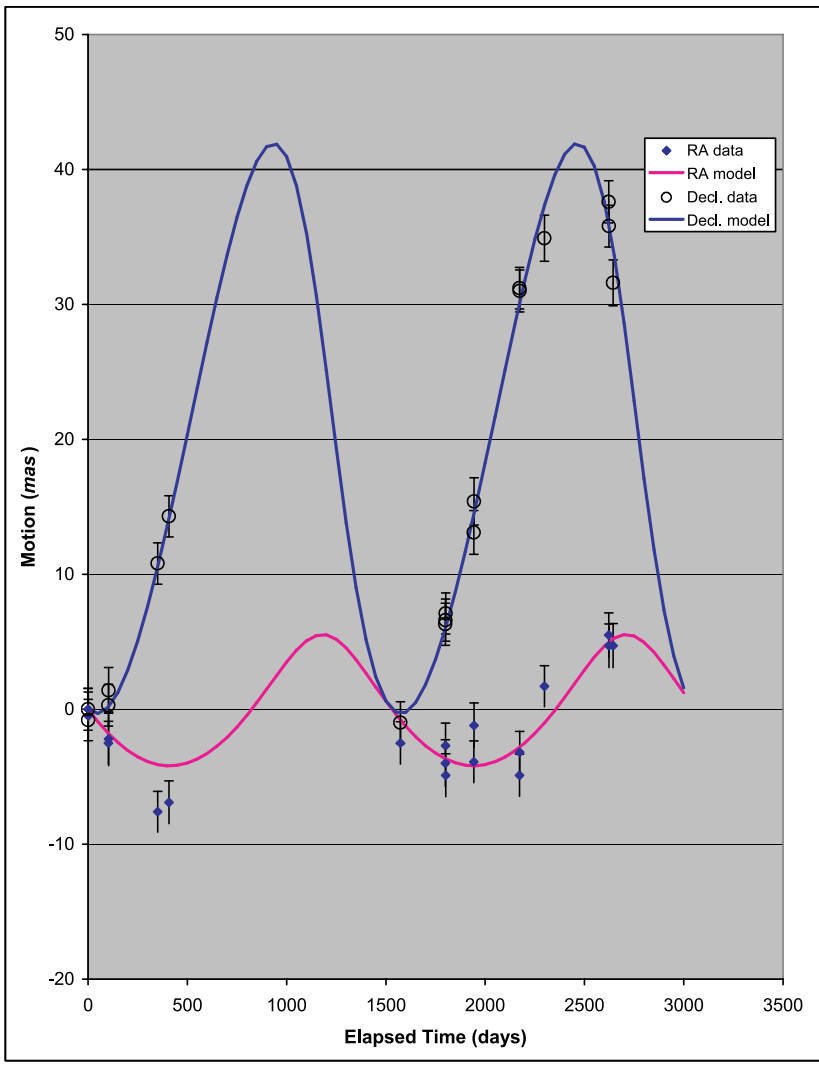

FIG. 1.-Astrometric motion of G78-28 measured with STEPS. The reference JD is $2,450,803.78$. The model has a orbital period $P=4.19 \mathrm{yr}$, eccentricity $e=0.26$, system mass $=0.57 M_{\odot}$, and semimajor axis $a=2.15$ AU.

in frames can suggest false accelerations of the target stars, limiting the accuracy of orbits with periods long compared to the observational baseline. However, the effect is negligible for STEPS, as the corrections are only hundredths of milliarcseconds for baselines of $\sim 10 \mathrm{yr}$.

G78-28 has a periodic astrometric signal after subtraction of parallax and proper motion from the total motion, indicating the presence of the companion, G78-28B. Figures 1 and 2 show the astrometric data superimposed on an orbit with an acceptable fit. Our error estimates comprise the uncertainty due to the Poisson errors derived from the standard error of the mean of a set of 1020 exposures each night added in quadrature to 1.5 mas systematic errors. The latter could be due to unmodeled real motions (e.g., other companions) or currently unmeasured instrumental errors. We use the Monte Carlo technique to determine the $1 \sigma$ confidence limits in our observed parameters via the method described in Lampton et al. (1976) for multiparameter estimation (see Pravdo et al. [2004] for further details). Table 2 lists the orbital parameters.

\subsubsection{GJ 231.1BC}

We observed GJ 231.1B (= G106-36, HD 43587B) over the same approximate span as G78-28, but starting one day earlier, JD 2,450,802.8. Table 1 also lists its previously known properties. The parallax value for the companion GJ 231.1A (an F9 V star) is $50 \pm 9.6$ mas (see footnote 1), consistent with the Reid et al. (1995) combined trigonometric and photometric parallax for GJ 231.1B of $50 \pm 10$ mas. The proper motion and position angle (P.A.) values in Table 1 are the mean values of the prior measurements listed, and the errors are the standard deviations. The STEPS absolute parallax is $55.2 \pm 1.0$ (Table 2 ). This 


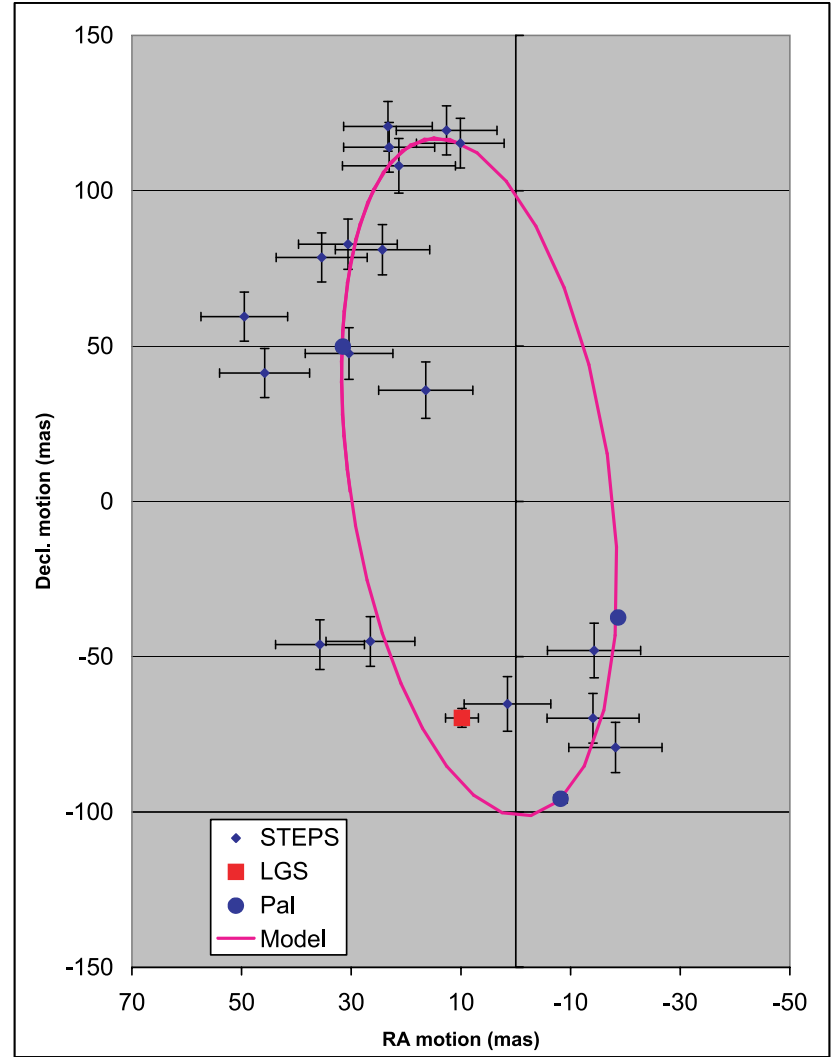

FIG. 2.-G78-28 astrometric data (points) superimposed on the orbital model (curve) with the parameters listed in the Fig. 1 caption. The data are from STEPS (black diamonds), LGSAO (red square), and Palomar AO (blue circles). The Palomar AO measurement errors are the size of the symbols.

and the measured proper motion are consistent with the prior results.

The astrometric fits to the GJ 231.1B motion indicate the presence of the companion GJ 231.1C. The residual or systematic error in the fits is 1.7 mas and arises in part from the variation with orbital phase in the shape of the point-spread function (PSF) of GJ 231.1BC. We now know (see $\S 2.2$ ) that this PSF contains a bright companion with a separation that is $\sim 1 / 3$ the size of the PSF of an unresolved source in good seeing. These variations add noise to the fitting process.

Figure 3 shows the allowed orbital periods for GJ 231.1BC versus system eccentricity. The minimum period is $\sim 25 \mathrm{yr}$. The period is $<200 \mathrm{yr}$ for eccentricity $e<0$.7. Figure 4 shows the data superposed on a possible orbit with $\sim 100$ yr period. The relatively short temporal baseline compared with the minimum period does not allow us to usefully restrict the dynamical mass. Figure 3 shows values in models for which the total mass is limited by the MLRs (§ 3.3).

\subsection{Laser Guide Star Adaptive Optics Imaging}

\subsubsection{Observations}

Observations of G78-28 and GJ 231.1B were taken on 2005 January 5 with the laser guide star adaptive optics (LGSAO) system at the Keck II telescope (Wizinowich et al. 2004) and the narrow camera mode of the Near-Infrared Camera 2 (NIRC2). The laser excites a region of sodium atoms at an altitude of roughly $90 \mathrm{~km}$, and the resulting emission is bright enough to allow for correction of the lower atmosphere by the rest of the AO train. The advent of LGSAO allows fainter objects to be observed with

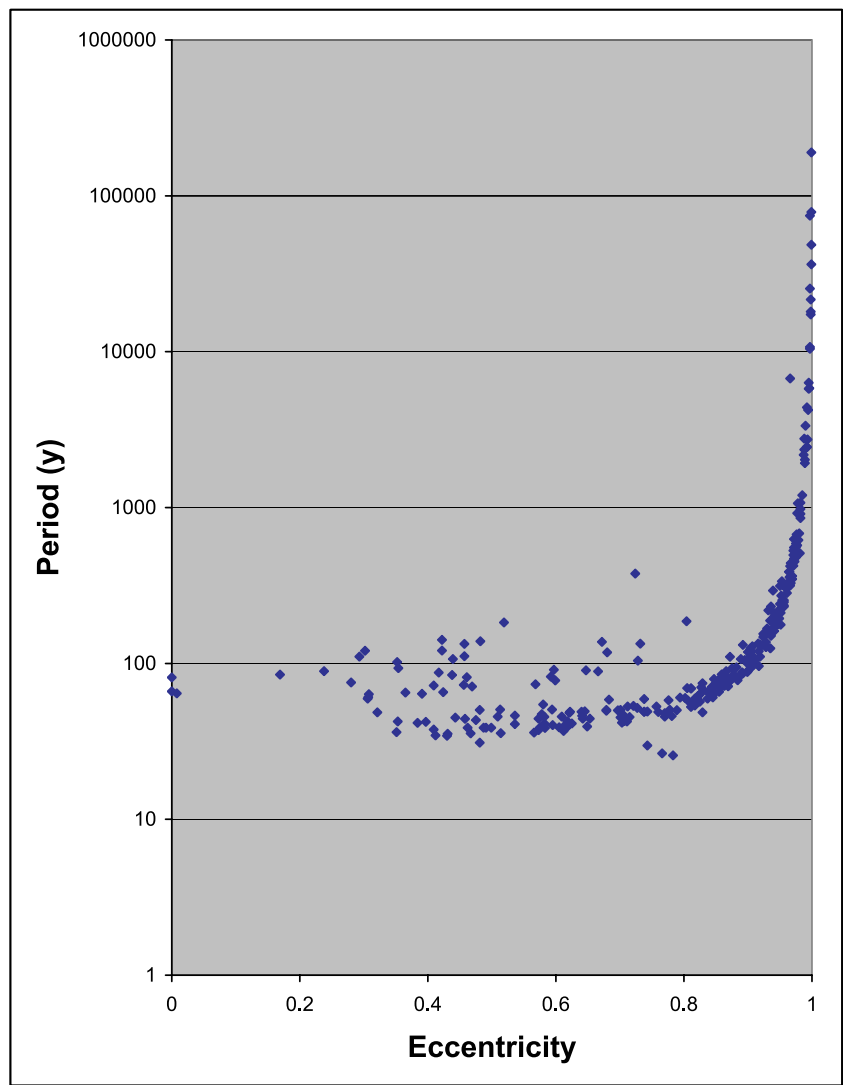

FIG. 3.-GJ 231.1BC allowed orbital periods vs. eccentricity. The total system mass is restricted to the value and uncertainty determined with the MLRs.

AO, because a bright star ( $V$ magnitude $\approx 13)$ is no longer necessary for high-order correction. However, since the laser and the observed sodium emission sample the same volume of air, loworder, tip-tilt correction requires another source. This is satisfied by a nearby, fainter star $(V$ magnitude $\approx 16$ ), because low-order correction requires fewer photons.

G78-28 was observed in the $J, H$, and $K p$ bands, and only the $H$-band data were used for astrometry (710 co-added images of $0.2 \mathrm{~s}$ each; see Fig. 5). The $K p$ band is centered at $2.124 \mu \mathrm{m}$ with a bandwidth of $0.351 \mu \mathrm{m} .{ }^{3}$ The binary separation was too small for the poor $J$-band correction to be useful. While the $K p$-band correction was excellent, the images were unfortunately taken in a mode that allowed the sky to rotate on the detector. This made accurate P.A. determination difficult. We obtained photometry in all three bands.

GJ 231.1B was observed in the $J(130$ co-added images of $0.5 \mathrm{~s}$ each) and $K p$ bands (550 co-added images of $0.5 \mathrm{~s}$ each; see Fig. 6 ); astrometry and photometry were determined for both. Even though the $J$-band data are less extensive, astrometry between the $J$ and $K p$ bands agrees to 0.5 mas separation and 0.20 P.A. We summarize the data from both sources in Table 3. Figure 2 shows the LGSAO data with the other data and the orbital model.

\subsubsection{Data Reduction}

G78-28A and B are partially resolved; B sits on a substantial gradient of the A PSF (Fig. 5). We estimated the value of the A PSF at the location of the B peak by measuring the value of the A PSF at several radial distances equal to the nominal separation of

\footnotetext{
${ }^{3}$ See http://alamoana.keck.hawaii.edu/inst/nirc2/Manual/ObserversManual html.
} 


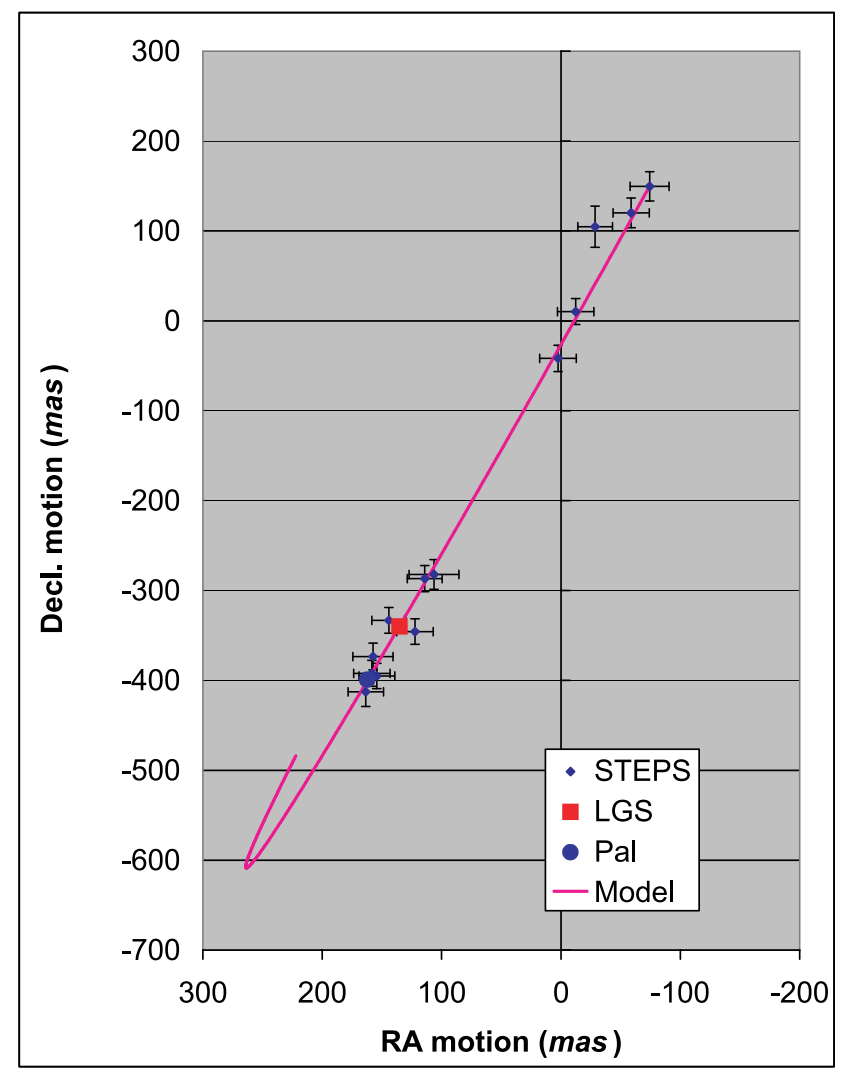

FIG. 4.-Possible orbit for GJ 2311.1BC. The data are from STEPS (black diamonds), LGSAO (red square), and Palomar AO (blue circles). The model is for an orbit with $P=102 \mathrm{yr}$, eccentricity $e=0.43$, system mass $=0.37 M_{\odot}$, and semimajor axis $a=15.6$ AU.

AB. GJ 231.1B and $\mathrm{C}$ are well resolved (Fig. 6), but $\mathrm{C}$ sits on a significant background due to the $\mathrm{AO}$ halo around $\mathrm{B}$.

The background was removed by taking advantage of the circular symmetry of the AO halo. We rotated the image of GJ 231.1BC about the peak of B and subtracted it from the nonrotated image. This resulted in positive and negative images of $\mathrm{C}$

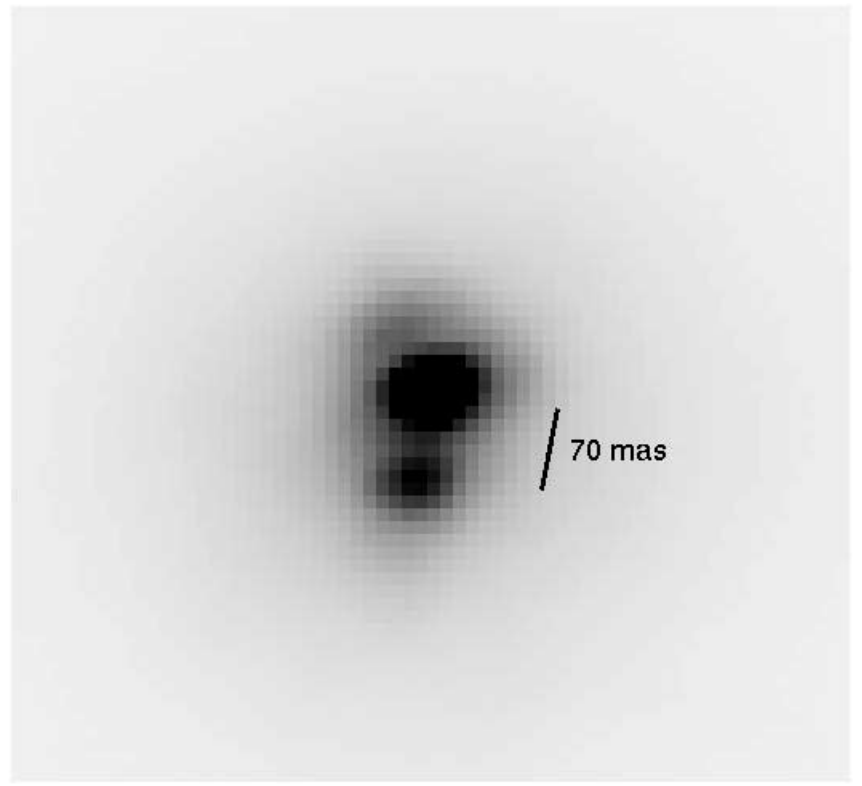

FIG. 5.-G78-28A and B in the $H$ band. North is up and east is to the left.

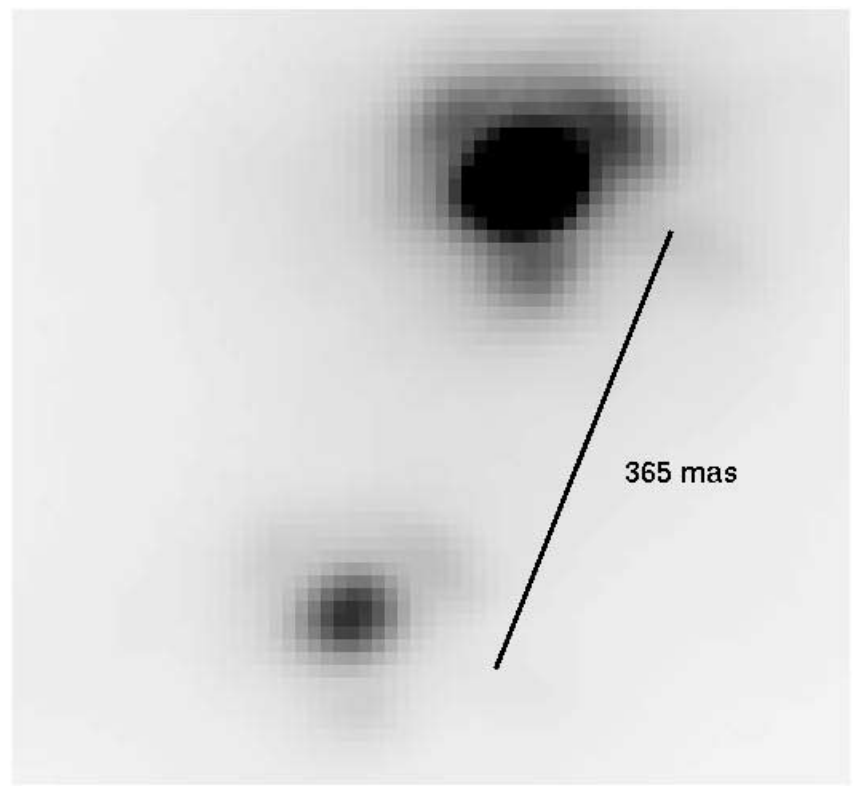

FIG. 6.-GJ 231.1B and $\mathrm{C}$ in the $K p$ band. North is up and east is to the left.

with a nominally flat background except for evidence of the AO side lobes close to the B image. The halo appears to be removed to $\sim 1 \%$ of the $\mathrm{B}$ peak.

We measured the separations and P.A.'s using the $K p$ images because the G78-28 and GJ 231.1 PSFs are similar. The PSFs in $J$ of the GJ 231.1 image are 22 times broader than for G78-28, making image subtraction problematic. The PSF fitting region was 80 mas. We fit the GJ 231.1B PSF to the core of the G7828A PSF while masking the pixels at and around the G78-28B image. Fitting parameters were position and amplitude. We then subtracted the shifted and scaled GJ 231.1B image from the G78-28A image, resulting in a nominally flat background clearly showing a positive peak at G78-28B and a negative peak at GJ 231.1C. Finally, we fit a spline to the residuals and measured the peak location. The measurements of the pixel scales are described in the Appendix.

\subsection{Palomar Adaptive Optics Imaging}

We observed G78-28 in the $H$ band with the Palomar 200 inch AO system (Troy et al. 2000) as part of a program to explore precision calibration of AO images with the application of nonredundant aperture-masking interferometry. The difficulties described in $\S 2.2 .2$ regarding the removal of the PSF and extraction of the astrometric and photometric measurements are inherent to the images produced by AO systems. The fluctuation of the unstable AO PSF limits the precision and sensitivity of AO observations. Substantial progress has been made in solving this

TABLE 3

LGSAO MEasurements

\begin{tabular}{|c|c|c|c|c|}
\hline Binary & Band & $d$ Mag & $\begin{array}{l}\text { Separation } \\
\quad(\text { mas })\end{array}$ & $\begin{array}{l}\text { P.A. } \\
\text { (deg) }\end{array}$ \\
\hline G78-28AB $\ldots$. & $J$ & $1.24 \pm 0.07$ & & \\
\hline G78-28AB & $H$ & $1.24 \pm 0.07$ & $70.4 \pm 2.5$ & $172 \pm 2$ \\
\hline 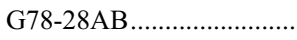 & $K p$ & $1.14 \pm 0.06$ & $\ldots$ & $\ldots$ \\
\hline 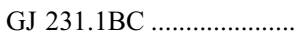 & $\mathrm{J}$ & $1.65 \pm 0.05$ & & \\
\hline GJ 231.1BC & $\mathrm{Kp}$ & $1.52 \pm 0.05$ & $366 \pm 3$ & $158 \pm 1$ \\
\hline
\end{tabular}

Note.—JD $=2,453,376.0$. 

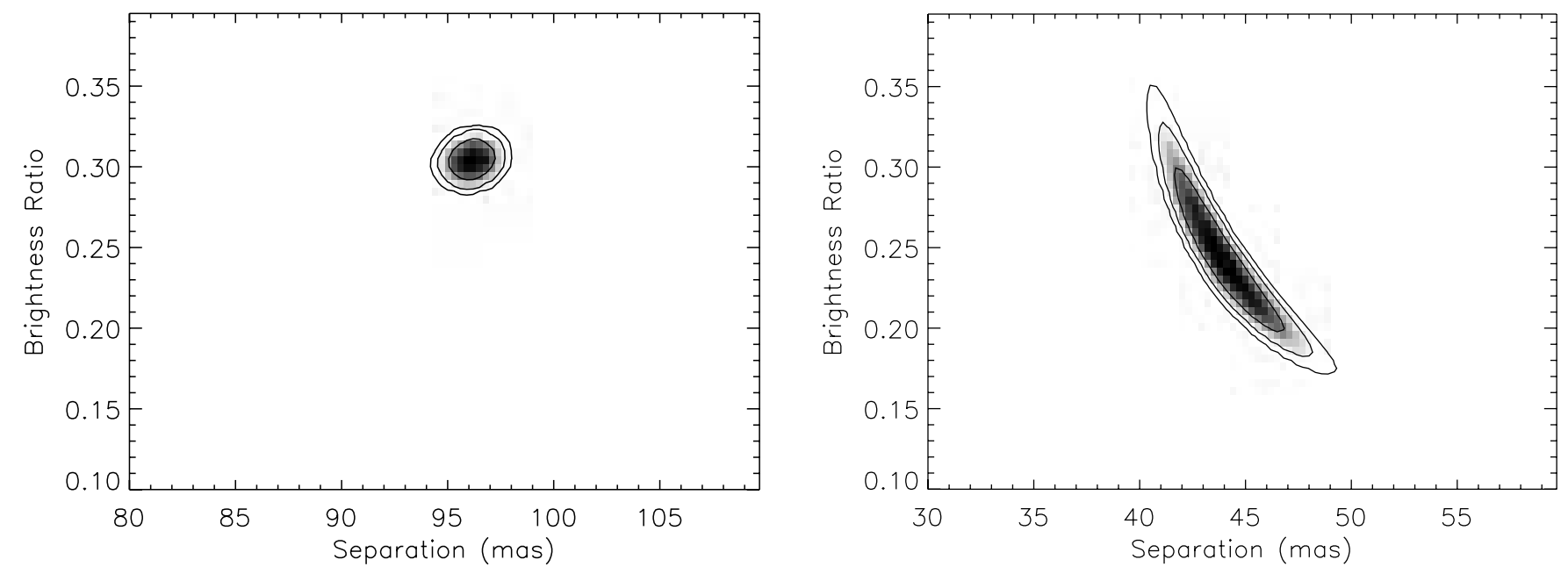

FIG. 7.-Correlation between the derived parameters of brightness ratio and separation for the Palomar AO observations of G78-28 on JD 2,453,779.7 (left) and $2,453,779.7$ (right). The contours show confidence regions of $90 \%, 99 \%$, and $99.9 \%$.

problem by exploiting simultaneous differential measurements in polarization (Apai et al. 2004; Potter 2003; Perrin et al. 2004) or wavelength (Close et al. 2005; Marois et al. 2005). These techniques are useful in cases in which there is a large differential signal, but do not address the generic problem of the fidelity of AO imaging.

An alternative approach to the exploitation of the coherent wave front provided by an $\mathrm{AO}$ system is the application of aperturemasking interferometry (Tuthill et al. 2000) instead of conventional imaging. For these observations, we placed a nine-hole mask in the Lyot stop of the PHARO camera (Hayward et al. 2001) with $50 \mathrm{~cm}$ projected hole diameters and a longest baseline of $415 \mathrm{~cm}$. The hole positions are chosen to maximize Fourier coverage and transmission while maintaining nonredundancy to preserve closure phase (Haniff et al. 1987; Readhead et al. 1988). The resulting interferogram is recorded in the image plane. This interferogram records all 36 pairwise fringes from the nine holes in the mask.

The advantages of this approach are several-fold. The AO PSF instability is a result of the fluctuations in the residual atmospheric phase and AO system calibration errors. The preservation of closure phase by the nonredundancy allows the use of self-calibration techniques (Cornwell 1989), thus rejecting these residual phase errors. The calibration problem is also simplified to a well-posed problem of calibrating the visibility of a single interferometer baseline at one time, rather than the ill-posed inverse problem of deconvolution of an image with an unknown PSF. Finally, an interferometric approach enables "superresolution" if the calibration is sufficiently accurate. In comparison with uncompensated aperture masking interferometry (Tuthill et al. 2000; Nakajima et al. 1989), AO provides stabilization of the fringes, enabling long integration times and therefore reaching to fainter targets.

The data are calibrated with observations of a nearby star. Care must be taken to select a source that is of similar brightness to both the $\mathrm{AO}$ wave front sensor operating in the red optical and the science camera operating in the infrared to ensure a comparable wave front correction and signal-to-noise ratio. The data are dark-subtracted, flat-fielded, and analyzed with a custom software pipeline written in IDL. The pipeline outputs a bispectrum in OIFITS format (Pauls et al. 2005). A binary model is fit to the bispectrum. Although the binary signal is apparent in visibility amplitude through a power spectrum, in practice we have found that the visibility amplitude calibration is poor, and superior re- sults are achieved with a fit to the closure phase alone. Presumably this is because the visibility amplitude calibration is susceptible to the same fluctuations in seeing and AO performance between source and calibrator that plague conventional imaging with $\mathrm{AO}$. For the observations reported here we neglect the visibility amplitude, and the model is fit to the phase of the bispectrum.

G78-28 was observed in four observing runs in 2003 December, 2005 September, 2005 December, and 2006 February. No fringes were detected in the 2005 September observations due to poor seeing. At $V$ magnitude 12.4, G78-28 is near the performance limit of the natural guide star $\mathrm{AO}$ system, requiring the $\mathrm{AO}$ system to operate at reduced bandwidth. The fringe stability is therefore a sensitive function of the atmospheric turbulence. Median closure phase scatter was $3.5,0.6$, and 1.2 in the 2003 December, 2005 September, and 2006 February data sets, respectively. The 2005 September (JD 2,453,632.9) data are in the best seeing, and fortuitously, at the largest separation. The bispectrum model fit typically had reduced $\chi^{2}>1$, attributed to a systematic effect. We added a systematic error to achieve $\chi^{2}=1$ and determine the confidence intervals. Starting in 2006 February, we increased the number of times that we cycled between source and calibrator, and this eliminated the need for the added error. The resulting final closure phase errors were 4.3 , 2.0 , and 1.2 on the three data sets.

The resulting extraction of astrometric parameters is a sensitive function of the orbital separation. In cases in which the binary is well resolved, the solution is unambiguous. On JD 2,453,779.7 the separation of 96 mas $(1.45 \lambda / D)$ is well resolved, and the resulting likelihood function is unambiguously fit with a unique separation and contrast (Fig. 7, left). However, on JD 2,453,632.9 the separation is only 41 mas $(0.62 \lambda / D)$. Even though this is well below the conventional resolution limit of the telescope, the binary is well detected, but there is degeneracy between the separation and contrast ratio. The likelihood contours shown in Figure 7 approximately define a locus of constant closure phase. For small separations and brightness ratios, closure phase is proportional to brightness ratio and separation cubed. Since the contrast ratio is well constrained by the better resolved observation, we adopt the JD 2,453,779.7 contrast ratio for the other observations. The resulting parameters are shown in Table 4 . The $H$-band magnitude difference, $1.285 \pm 0.023$, is consistent with the LGSAO result at better than $1 \sigma$. Figure 2 shows the Palomar AO orbital data with the other data and the orbital model. 
TABLE 4

Palomar aO Measurements

\begin{tabular}{|c|c|c|c|c|c|}
\hline Binary & Julian Date & Band & $d$ Mag & $\begin{array}{c}\text { Separation } \\
\text { (mas) }\end{array}$ & $\begin{array}{l}\text { P.A. } \\
\text { (deg) }\end{array}$ \\
\hline G78-28AB.. & $2,453,004.8$ & $H$ &.$^{a}$ & $58.9 \pm 1.3$ & $32.3 \pm 1.1$ \\
\hline G78-28AB.. & $2,453,632.9$ & $H$ & $1.285 \pm 0.023$ & $96.1 \pm 1.1$ & $184.9 \pm 0.7$ \\
\hline G78-28AB. & $2,453,779.7$ & $H$ & $\ldots{ }^{\mathrm{a}}$ & $41.8 \pm 0.6$ & $206.6 \pm 0.9$ \\
\hline GJ 231.1BC. & $2,453,779.7$ & $H$ & $1.64 \pm 0.06$ & 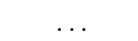 & \\
\hline 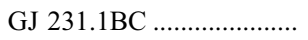 & $2,453,779.7$ & $K_{s}$ & $1.53 \pm 0.04$ & $431 \pm 4$ & $157.9 \pm 0.7$ \\
\hline
\end{tabular}

${ }^{\text {a }}$ Fixed at the G78-28 JD 2,453,632.9 value.

GJ 231.1B was observed on JD 2,453,779.7. It was well resolved and detected with conventional AO imaging. At $V=$ $13.3 \mathrm{mag}$ it is near the performance limit of the natural guide star AO system. Due to the fluctuating wave front quality, we found it necessary to take about 100 short-exposure images in the $H$ and $K_{s}$ bands and only use the images with the highest Strehl ratio to extract the brightness ratios. The separation, P.A., and delta magnitudes for $H$ and $K_{s}$ are shown in Table 4. The $K$-band delta magnitudes from LGS and Palomar AO are consistent with each other. The positional data are plotted in Figure 4.

\section{DISCUSSION}

\section{1. $G 78-28 A B$}

The G78-28AB system is $18.4 \pm 0.3 \mathrm{pc}$ from the Sun. The composite light was spectrally classified as a dwarf M3 (e.g., Reid et al. 1995). It is a moderately active X-ray star (Hünsch et al. 1999) with about $1 / 3$ the X-ray luminosity of GJ $802 \mathrm{Ab}$, a newly discovered system with a close companion (Pravdo et al. 2005a). Although there are only small numbers of identified close binaries such as these, a correlation between $\mathrm{M}$ dwarfs with close companions and X-ray emission may emerge as the binarity of heretofore "single" $\mathrm{M}$ dwarfs is discovered. Its $(U, V, W)$ space velocity measured by Reid et al. (1995) is consistent with its being a member of their local volume-complete sample of $M$ dwarfs.

We measure the dynamic total mass in the astrometric fits to be $0.60 \pm 0.09 M_{\odot}$. We also find that $\alpha / a=f-\beta=0.195 \pm$ 0.025 , where $\alpha$ is the photocentric axis, $a$ is the semimajor axis, $f$ is the secondary mass fraction, and $\beta$ is the secondary light fraction. From the $M_{V}$ of the system and the visible MLRs we calculate $f$ and $f-\beta$ with $\beta$ as the independent variable. The measured $f-\beta$ corresponds to a total mass $<0.51 M_{\odot}$ (eq. [5b] of Henry \& McCarthy [1993]; eq. [7] of Henry et al. [1999]) or $<0.57 M_{\odot}$ (Delfosse et al. 2000), where the uncertainty in the upper limits is $0.11 M_{\odot}$ (from the dispersion given in Henry \& McCarthy 1993) and the lower limits are not determinable because the secondary mass becomes too small to be in the applicable range of MLRs.

TABLE 5

Component Magnitudes

\begin{tabular}{crrrr}
\hline \hline Band & \multicolumn{1}{c}{ G78-28A } & \multicolumn{1}{c}{ G78-28B } & \multicolumn{1}{c}{ GJ 231.1B } & \multicolumn{1}{c}{ GJ 231.1C } \\
\hline$M_{V} \ldots \ldots \ldots$. & $11.13 \pm 0.05$ & $12.98 \pm 0.27$ & $12.05 \pm 0.07$ & $14.90 \pm 0.21$ \\
$M_{J}^{\mathrm{a}} \ldots \ldots \ldots .$. & $7.40 \pm 0.03$ & $8.64 \pm 0.07$ & $8.01 \pm 0.02$ & $9.66 \pm 0.05$ \\
$M_{H}^{\mathrm{a}} \ldots \ldots \ldots$. & $6.83 \pm 0.03$ & $8.12 \pm 0.07$ & $7.48 \pm 0.04$ & $9.12 \pm 0.06$ \\
$M_{K}^{\mathrm{a}} \ldots \ldots \ldots$. & $6.59 \pm 0.02$ & $7.73 \pm 0.06$ & $7.22 \pm 0.01$ & $8.73 \pm 0.05$ \\
\hline
\end{tabular}

${ }^{\text {a }}$ Based on 2MASS and Tables 3 and 4 .
Table 5 shows the magnitudes derived for both components in four bands. We derive the delta $V$ between G78-28A and B of $1.85 \pm 0.28 \mathrm{mag}$ from the measured $M_{J H K}$ (Tables 1, 3, and 4) and the color-magnitude relationships for $\mathrm{M}$ dwarfs (eqs. [1a][1c] of Henry \& McCarthy 1993). The delta $V$ magnitude corresponds to $0.12 \leq \beta \leq 0.19$, but the measured $f-\beta$ can further constrain it depending on the MLR. For Henry \& McCarthy (1993), $0.12 \leq \beta \leq 0.17$. The magnitude range alone constrains the total mass to be $0.508 \pm 0.002 M_{\odot}$ and $f=0.33 \pm 0.01$. For Delfosse et al. (2000), $\beta$ is not further constrained, and the total mass is $0.554 \pm 0.009 M_{\odot}$ and $f=0.37 \pm 0.02$. The predictions of the MLRs are consistent, once we consider the mass dispersions (Henry \& McCarthy 1993), viz., $0.11 M_{\odot}$ for the sum. A further detailed discussion of the system masses is in $\S 3.3$, where we estimate a consistent value and error based on the MLRs in several bands.

With $V-K$ values of $4.54 \pm 0.05$ and $5.25 \pm 0.28$, the inferred spectral types are M2.5 and M4, for A and B, respectively, within a couple of tenths of a subtype (e.g., Leggett 1992).

\subsection{GJ 231.1BC}

GJ 231.1 A and B are a parallax and proper motion pair (Poveda et al. 1994) consisting of an F9 V primary and an M3.5 V secondary (Reid et al. 1995) at a distance of $18.1 \pm 0.3 \mathrm{pc}$. The secondary is now shown to consist of two stars, B and C. The large separation of $\mathrm{B}$ and $\mathrm{C}$ indicates a longer period than our observing baseline and correspondingly larger uncertainties in the orbital parameters. It is clear from our two imaging observations that $\mathrm{B}$ and $\mathrm{C}$ are also a parallax and proper motion pair, since their relative separation changes by much less than the proper motion during the interval between the observations. The orbit is nearly edge-on (Fig. 4). GJ $231.1 \mathrm{BC}$ may be relatively inactive, with no reported $\mathrm{H} \alpha$ or X-ray emission. It is also a member of the local sample based on its distance, $18.1 \mathrm{pc}$, and space velocities (Reid et al. 1995). It has roughly solar metallicity, $[\mathrm{Fe} / \mathrm{H}]=-0.02 \pm 0.04$ (Bonfils et al. 2005).

We again use the magnitude-color relationships to estimate that between the components, delta $V=2.85 \pm 0.21 \mathrm{mag}$. Table 5 shows the component magnitudes. The $V-K$ magnitudes are $4.83 \pm 0.09$ for $\mathrm{GJ} 231.1 \mathrm{~B}$ and $6.17 \pm 0.21$ for $\mathrm{C}$, resulting in types of M3.5 for B and M5 for C. The masses for this system are not well constrained by the astrometry, but we use the MLRs again for estimates in the following section.

\subsection{Mass Estimation}

In the cases of unresolved astrometric systems in which the secondary contributes significantly to the total light, such as the subjects of this paper, we do not observe the secondary mass fraction, $f$, directly, but rather the quantity $f-\beta$. When we resolve the systems with imaging we measure the secondary light fraction, 
TABLE 6

G78-28AB Masses Derived from MLRs

\begin{tabular}{|c|c|c|c|c|c|c|}
\hline \multirow[b]{2}{*}{ BAND } & \multicolumn{3}{|c|}{$\operatorname{MASS}^{\mathrm{a}}\left(M_{\odot}\right)$} & \multicolumn{3}{|c|}{$\operatorname{MASs}^{\mathrm{b}}\left(M_{\odot}\right)$} \\
\hline & G78-28A & G78-28B & Total & G78-28A & G78-28B & Total \\
\hline V & $0.34 \pm 0.08$ & $0.17 \pm 0.07$ & $0.51 \pm 0.11$ & $0.35 \pm 0.01$ & $0.20 \pm 0.02$ & $0.55 \pm 0.03$ \\
\hline J & $0.38 \pm 0.10$ & $0.18 \pm 0.03$ & $0.56 \pm 0.11$ & $0.35 \pm 0.02$ & $0.21 \pm 0.01$ & $0.55 \pm 0.03$ \\
\hline 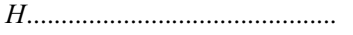 & $0.34 \pm 0.08$ & $0.17 \pm 0.04$ & $0.51 \pm 0.08$ & $0.35 \pm 0.02$ & $0.19 \pm 0.01$ & $0.54 \pm 0.03$ \\
\hline 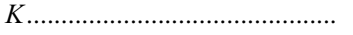 & $0.34 \pm 0.07$ & $0.18 \pm 0.04$ & $0.52 \pm 0.08$ & $0.35 \pm 0.02$ & $0.20 \pm 0.01$ & $0.55 \pm 0.02$ \\
\hline$V-K$ & $\ldots$ & .. & ... & $0.35 \pm 0.01$ & $0.20 \pm 0.03$ & $0.50 \pm 0.04$ \\
\hline 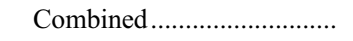 & $0.35 \pm 0.08$ & $0.18 \pm 0.04$ & $0.53 \pm 0.09$ & $0.35 \pm 0.02$ & $0.20 \pm 0.02$ & $0.54 \pm 0.03$ \\
\hline
\end{tabular}

a Based on Henry \& McCarthy (1993).

${ }^{\mathrm{b}}$ Based on Delfosse et al. (2000).

$\beta$, but since the components generally have different colors, the measurement of $\beta$ in the IR does not unambiguously yield $\beta$ in $V$, where required. However, as shown in $\S 3.1$, the color-magnitude relationships can be used to extrapolate from $H$ to $V$. These two systems illustrate our increasing abilities to make mass estimates. For G78-28 we use the astrometry and the imaging to make estimates for the total and component masses. Table 6 shows the estimates based on the measured parallax, apparent magnitudes, the measured $J H K$ ratios, the inferred $V$ ratio, and two current MLRs (eqs. [2]-[5] of Henry \& McCarthy 1993 [HMLR]; Delfosse et al. 2000 [DMLR]). The uncertainties in HMLR consist of both the dispersion that they provide and our measurement errors, while those in DMLR are only the latter. There is good agreement within each MLR among the masses determined from the visible and near-IR colors. The two MLRs agree well for the mass of the primary, $M_{A}=0.35 \pm 0.08 M_{\odot}$, but DMLR consistently predicts higher masses for the secondary, which we adopt, $M_{B}=$ $0.18 \pm 0.04 M_{\odot}$. However, the estimates largely overlap.

The dynamical mass ( $\S 3.1$ ) and the masses derived from the MLRs overlap, but the mean of the dynamical mass is higher. The intersection of the two methods yields our adopted total mass estimate of $0.565 \pm 0.055 M_{\odot}$. Taking the derived values for $f(\S 3.1)$, we get $M_{B}=0.195 \pm 0.021$, consistent with the dynamical estimate and the two MLRs. Then, $M_{A}=0.370 \pm$ 0.034 . We also estimate the spectral types from the mass-type relation in Kirkpatrick \& McCarthy (1994). The types are M2 and M4 for A and B, respectively. Note that both the colors and the mass-type relation yield an earlier type for G78-28A by $0.5-1$ subtype than its prior value.

We perform a similar analysis for GJ 231.1BC and show the results in Table 7. Again, although the MLR estimates overlap, the masses derived from HMLR are consistently $0.01-0.02 M_{\odot}$ smaller than those derived from DMLR. For this system, $M_{B}=$ $0.25 \pm 0.06 M_{\odot}$ and $M_{C}=0.12 \pm 0.02 M_{\odot}$. The spectral types are M3 and M5 for B and C, respectively, from the mass-type relation (Kirkpatrick \& McCarthy 1994). As in the case of G7828 , the mass-type relation yields a subtype that is $\sim 0.5$ earlier than the color-derived type for the primary, but agrees well for the secondary.

At the current level of knowledge and because of the intrinsic scatter of luminosities and masses due to stellar properties such as age and metallicity, the fact that systems such as G78-28AB and GJ 231.1BC are binary has little impact on the estimated masses (or spectral types) of the primaries alone. For example, the mass estimate for G78-28A decreases as calculated in HMLR by only $\sim 0.03 M_{\odot}$, within the error, because of the small decrease in luminosity of this binary component. Similarly, GJ 231.1B decreases by only $\sim 0.01 M_{\odot}$. However, the total masses of the systems change more dramatically, from 0.42 to $0.52 M_{\odot}$ for $\mathrm{G} 78-28 \mathrm{AB}$, and from 0.29 to $0.37 M_{\odot}$ for GJ 231.1BC, even if we use the larger mass estimates for the single stars derived from the near-IR MLRs. If all the light came from a single star in both systems, the near-IR MLR mass estimates would be consistently higher than those due to the visible for both HMLR and DMLR. This is because the primary accounts for a smaller fraction of the light in the IR than in the visible. Therefore the IR mass estimate drops further than the visible mass estimate for the primary. Conversely, if the star is incorrectly believed to be single, then there is more erroneously assigned light in the IR than in the visible, leading to a higher mass estimate in the IR. This apparent discrepancy in the masses derived from MLRs is largely removed with the discoveries of binarity (Tables 6 and 7).

Our derived masses for the four components agree well with theoretical model estimates. Figure 8 shows our stellar $M_{K}$ and

TABLE 7

GJ 231.1BC Masses Derived from MLRs

\begin{tabular}{|c|c|c|c|c|c|c|}
\hline \multirow[b]{2}{*}{ BAND } & \multicolumn{3}{|c|}{$\operatorname{MAsS}^{\mathrm{a}}\left(M_{\odot}\right)$} & \multicolumn{3}{|c|}{$\operatorname{MAss}^{\mathrm{b}}\left(M_{\odot}\right)$} \\
\hline & GJ 231.1B & GJ $231.1 \mathrm{C}$ & Total & GJ 231.1B & GJ $231.1 \mathrm{C}$ & Total \\
\hline 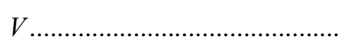 & $0.25 \pm 0.05$ & $0.11 \pm 0.02$ & $0.37 \pm 0.06$ & $0.28 \pm 0.01$ & $0.12 \pm 0.02$ & $0.41 \pm 0.03$ \\
\hline J & $0.26 \pm 0.07$ & $0.12 \pm 0.02$ & $0.38 \pm 0.09$ & $0.27 \pm 0.01$ & $0.13 \pm 0.05$ & $0.39 \pm 0.02$ \\
\hline H. & $0.24 \pm 0.05$ & $0.12 \pm 0.02$ & $0.36 \pm 0.07$ & $0.27 \pm 0.01$ & $0.13 \pm 0.01$ & $0.40 \pm 0.02$ \\
\hline K. & $0.24 \pm 0.05$ & $0.12 \pm 0.02$ & $0.36 \pm 0.07$ & $0.26 \pm 0.01$ & $0.13 \pm 0.01$ & $0.38 \pm 0.01$ \\
\hline$V-K$ & & & & $0.27 \pm 0.01$ & $0.13 \pm 0.01$ & $0.40 \pm 0.02$ \\
\hline 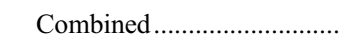 & $0.25 \pm 0.06$ & $0.12 \pm 0.02$ & $0.37 \pm 0.07$ & $0.27 \pm 0.01$ & $0.13 \pm 0.02$ & $0.40 \pm 0.02$ \\
\hline
\end{tabular}

${ }^{\text {a }}$ Based on Henry \& McCarthy (1993).

b Based on Delfosse et al. (2000). 


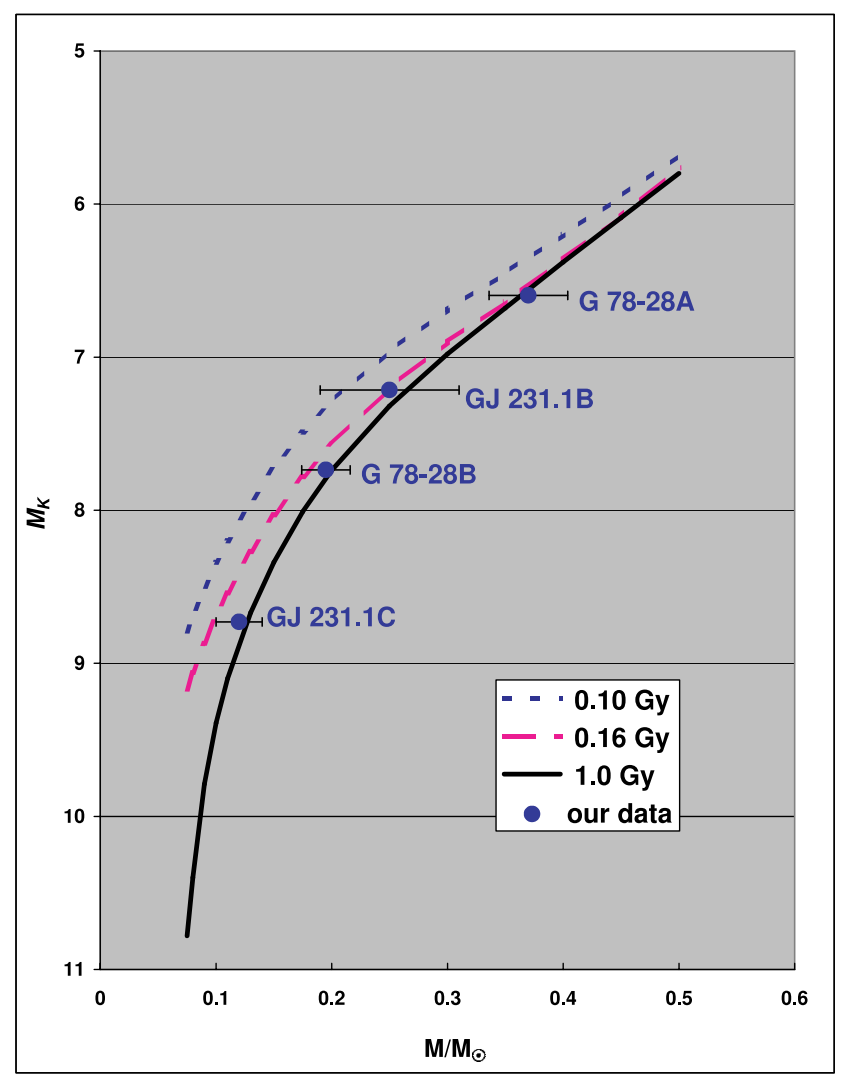

FIG. 8.- Plot of our data and the models of Barraffe et al. (1998). The models have solar metallicity with ages $t=10^{8} \mathrm{yr}$ (dotted line), $t=1.6 \times 10^{8} \mathrm{yr}$ (dashed line), and $t=10^{9} \mathrm{yr}$ (solid line). Our data points are labeled.

mass values on a plot with models of Barraffe et al. (1998). The data are consistent with models with solar or lower metallicity $([\mathrm{M} / \mathrm{H}]=0$ or -0.5$)$ and age $t \geq 1.6 \times 10^{8}$ yr. The models do not provide an age upper limit, since they are not distinguishable for $t>10^{9} \mathrm{yr}$ and these parameters.

\section{SUMMARY}

We have discovered two new low-mass binary systems with measurements of their astrometric motions. Our follow-up imaging observations resolved the systems in the near-IR. With the combined data we have improved mass estimates for four additional low-mass stars. Paths for further improvements are to lower the dispersion in the MLRs by using discoveries such as these to form larger homogeneous samples as their inputs, measure delta $V$ independently, and sharpen our knowledge of the orbit by extending the baseline of astrometric observations or otherwise increasing their accuracy.

The research described in this paper was performed in part by the Jet Propulsion Laboratory, California Institute of Technology, under contract with the National Aeronautics and Space Administration. We performed observations at Caltech's Palomar Observatory and Keck Observatory and acknowledge the assistance of the staff. We thank I. Baraffe and G. Chabrier for their assistance. This research has made use of the NASA/IPAC Infrared Science Archive, which is operated by the Jet Propulsion Laboratory, California Institute of Technology, under contract with the National Aeronautics and Space Administration. This research has made use of the SIMBAD database, operated at CDS, Strasbourg, France, and of NASA's Astrophysics Data System Abstract Service. This publication makes use of data products from the Two Micron All Sky Survey, which is a joint project of the University of Massachusetts and the Infrared Processing and Analysis Center/California Institute of Technology, funded by the National Aeronautics and Space Administration and the National Science Foundation. M. I. acknowledges Michelson Fellowship support from the Michelson Science Center and the NASA Navigator Program.

\section{APPENDIX}

\section{PLATE SCALE}

The LGSAO system provides a unique opportunity to determine plate scales for NIRC2. The core of the globular cluster M5 was observed on the night of 2005 April 30. A total of $400 \mathrm{Kp}$-band, co-added images of $0.2 \mathrm{~s}$ each were taken in the narrow and wide cameras. The images were dithered, and A. Bouchez's IDL routine nirc2warp.pro was used to remove known distortion from the NIRC2 detector ${ }^{4}$ before the images were combined into mosaics. The resulting mosaics are roughly $15^{\prime \prime}$ and $61^{\prime \prime}$ across for the narrow and wide cameras, respectively. The positions of 17 stars were compared between mosaics from both camera modes as well as from the Wide Field Planetary Camera 2 (WFPC2) aboard the Hubble Space Telescope. The stars span the area of the mosaics. The public, $96 \mathrm{~s}$ WFPC2 image was taken by F. R. Ferraro (proposal 6607) in the F555W filter on 1997 July 26 and retrieved using the Multimission Archive at Space Telescope. ${ }^{5}$ The four bright stars in the center of the fields were not used, as they saturated the NIRC2 detector.

In the WFPC2 image, the core of M5 lies on the high-resolution Planetary Camera chip. Holtzman et al. (1995) derive the Planetary Camera pixel scale as 45.54 mas pixel $^{-1}$ from observation of "several dozen" stars in the core of the globular cluster $\omega$ Cen, and they calculate the pixel scale as 45.55 mas pixel $^{-1}$ from observation of 11 stars in the globular cluster M67. No errors are given for their pixel scale; we therefore assume a Planetary Camera pixel scale of $45.545 \pm 0.005$ mas pixel $^{-1}$.

Because of the higher resolution of the NIRC2 mosaics, we assume that stellar centroiding is more accurate than in the Planetary Camera image. Therefore, we treat the NIRC2 mosaics as our reference images (described below). We fit second-order polynomials, in both $x$ - and $y$-axes, to the stellar positions between each reference image and the Planetary Camera image. We allow the pixel scales in the $x$ - and $y$-directions to differ from each other. Our transformations are given by

$$
\begin{gathered}
x^{\prime}=a x^{2}+b x+c y^{2}+d y+e x y+f, \\
y^{\prime}=g y^{2}+h x+i y^{2}+j y+k x y+l,
\end{gathered}
$$

\footnotetext{
${ }_{5}^{4}$ See http://www2.keck.hawaii.edu/optics/lgsao/software/nirc2warp.pro.

5 See http://archive.stsci.edu.
} 
TABLE 8

NIRC2 Pixel ScAles

\begin{tabular}{lcr}
\hline \hline NIRC2 Mode & $\begin{array}{c}x \text { Pixel Scale } \\
(\mathrm{mas})\end{array}$ & \multicolumn{1}{c}{$\begin{array}{c}y \text { Pixel Scale } \\
(\mathrm{mas})\end{array}$} \\
\hline Narrow …...... & $9.982 \pm 0.024$ & $9.958 \pm 0.012$ \\
Wide ............. & $39.905 \pm 0.076$ & $39.862 \pm 0.019$ \\
\hline
\end{tabular}

where the primed coordinates are stellar positions in the Planetary Camera image and the unprimed coordinates are positions in the NIRC2 reference images. Having accurate centroids for stars in the reference images is necessary for accurate transformations. The relative linear scalings, in the $x$ - and $y$-directions, between each NIRC2 mosaic and the Planetary Camera image are thus

$$
\begin{aligned}
& m_{x}=\sqrt{\left(\frac{\partial x^{\prime}}{\partial x}\right)^{2}+\left(\frac{\partial x^{\prime}}{\partial y}\right)^{2}}, \\
& m_{y}=\sqrt{\left(\frac{\partial y^{\prime}}{\partial x}\right)^{2}+\left(\frac{\partial y^{\prime}}{\partial y}\right)^{2}} .
\end{aligned}
$$

Because of the second-order terms in equations (1) and (2), relative scaling between the reference and Planetary Camera images is not independent of stellar position. This is due to errors in the mosaicking process as well as residual distortion present in the detector. These errors limit the accuracy to which pixel scale can be measured; observing a denser star field, for example, will not increase the accuracy of this measurement. The $x$ - and $y$-pixel scales for the NIRC2 detector are therefore given by

$$
\begin{aligned}
& s_{x}=\bar{m}_{x} s_{x}^{\prime}, \\
& s_{y}=\bar{m}_{y} s_{y}^{\prime} .
\end{aligned}
$$

Note that $\bar{m}$ represents the mean relative scaling calculated from all 17 stellar positions in the reference image. The primed quantities are on the opposite sides of the transformation quantities between equations (1)-(5) and (2)-(6); this is because the units of equations (1) and (2) are in pixels, while the units of equations (5) and (6) are per pixel. Uncertainties in pixel scale are calculated according to

$$
\begin{gathered}
\sigma_{s_{x}}=\sqrt{\left(\sigma_{m_{x}} s_{x}^{\prime}\right)^{2}+\left(\bar{m}_{x} \sigma_{s_{x}^{\prime}}\right)^{2}}, \\
\sigma_{s_{y}}=\sqrt{\left(\sigma_{m_{y}} s_{y}^{\prime}\right)^{2}+\left(\bar{m}_{y} \sigma_{s_{y}^{\prime}}\right)^{2}},
\end{gathered}
$$

where $\sigma_{m}$ represents the standard deviation of the relative scaling.

The P.A.'s of each pair of stars were compared between mosaics, and this allows the net rotation between each pair of mosaics to be determined. Since the header keywords for each mosaic were used to determine north on the detectors, the net rotation represents the accuracy of those keywords. Table 8 lists the $x$ - and $y$-pixel scales for both the NIRC 2 narrow and wide cameras, and Table 9 compares these with values in the literature. The net pixel scales in this work comprise the mean of the $x$-and $y$-pixel scales, and the error is half

\begin{tabular}{|c|c|c|}
\hline Group & $\begin{array}{c}\text { Narrow } \\
\left(\text { mas pixel }^{-1}\right)\end{array}$ & $\begin{array}{c}\text { Wide } \\
\left(\text { mas pixel }^{-1}\right)\end{array}$ \\
\hline Ghez et al..................... & $9.93 \pm 0.05$ & . \\
\hline König et al. ................. & $9.942 \pm 0.500$ & $\ldots$ \\
\hline Roe et al....................... & $9.95 \pm 0.02$ & $\ldots$ \\
\hline This work ...................... & $9.970 \pm 0.012$ & $39.884 \pm 0.039$ \\
\hline
\end{tabular}
the quadrature addition of the $x$ - and $y$-pixel scale errors. Finally, Table 10 shows the net rotation between each pair of mosaics.

Note that our $x$ - and $y$-pixel scales fall within the error bounds from the literature. Our narrow camera $x$ - and $y$-pixel scales agree to $1 \sigma$.

TABLE 9

Comparison to Literature 
TABLE 10

Accuracy of Rotation Keywords

\begin{tabular}{|c|c|}
\hline Pair of Mosaics & $\begin{array}{l}\text { Net Rotation } \\
\text { (deg) }\end{array}$ \\
\hline Narrow to wide.... & $0.026 \pm 0.087$ \\
\hline 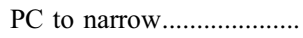 & $0.133 \pm 0.074$ \\
\hline 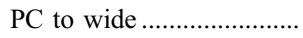 & $0.158 \pm 0.089$ \\
\hline
\end{tabular}

\section{REFERENCES}

Apai, D., Pascucci, I., Brandner, W., Henning, T., Lenzen, R., Potter, D. E. Lagrange, A.-M., \& Rousset, G. 2004, A\&A, 415, 671

Barraffe, I., Chabrier, G., Allard, F., \& Hauschmildt, P. H. 1998, A\&A, 337, 403

Bonfils, X., Delfosse, X., Udry, S., Santos, N. C., Forveille, T., \& Ségransan, D. 2005, A\&A, 442, 635

Close, L. M., et al. 2005, Nature, 433, 286

Cornwell, T. J. 1989, Science, 245, 263

Delfosse, X., Forveille, T., Ségransan, D., Beuzit, J.-L., Udry, S., Perrier, C., \& Mayor, M. 2000, A\&A, 364, 217

Gliese, W., \& Jahreiss, H. 1991, in The Astronomical Data Center CD-ROM: Selected Astronomical Catalogs, Vol. 1, ed. L. E. Brotzmann \& S. E. Gesser (Greenbelt: GSFC)

Haniff, C. A., Mackay, C. D., Titterington, D. J., Sivia, D., \& Baldwin, J. E. 1987, Nature, 328, 694

Hayward, T. L., Brandl, B., Pirger, B., Blacken, C., Gull, G. E., Schoenwald, J., \& Houck, J. R. 2001, PASP, 113, 105

Henry, T. J., \& McCarthy, D. W., Jr. 1993, AJ, 106, 773

Henry, T. J., et al. 1999, ApJ, 512, 864

Holtzman, J., et al. 1995, PASP, 107, 156

Hünsch, M., Schmitt, J. H. M. M., Sterzik, M. F., \& Voges, W. 1999, A\&AS, 135,319

Kirkpatrick, J. D., \& McCarthy, D. W., Jr. 1994, AJ, 107, 333

Lampton, M., Margon, B., \& Bowyer, S. 1976, ApJ, 208, 177

Leggett, S. K. 1992, ApJS, 82, 351

Lepine, S., et al. 2005, AJ, 129, 1483

Luyten, W. J. 1979, Catalogue of Stars with Proper Motions Exceeding 0".5 Annually (LHS) (2nd ed.; Minneapolis: Univ. Minnesota)
Marois, C., Doyon, R., Nadeau, D., Racine, R., Riopel, M., Vallée, P., \& Lafrenière, D. 2005, PASP, 117, 745

Nakajima, T., Kulkarni, S. R., Gorham, P. W., Ghez, A. M., Neugebauer, G., Oke, J. B., Prince, T. A., \& Readhead, A. C. S. 1989, AJ, 97, 1510

Pauls, T. A., Young, J. S., Cotton, W. D., \& Monnier, J. D. 2005, PASP, 117, 1255 Perrin, M. D., Graham, J. R., Kalas, P., Lloyd, J. P., Max, C. E., Gavel, D. T., Pennington, D. M., \& Gates, E. L. 2004, Science, 303, 1345

Potter, D. E. 2003, Ph.D. thesis, Univ. Hawai'i

Poveda, A., Herrera, M. A., Allen, C., Cordero, G., \& Lavalley, C. 1994, Rev. Mex. AA, 28, 43

Pravdo, S. H., Shaklan, S. B., Henry, T. J., \& Benedict, B. F. 2004, ApJ, 617, 1323

Pravdo, S. H., Shaklan, S. B., \& Lloyd, J. 2005a, ApJ, 630, 528

Pravdo, S. H., Shaklan, S. B., Lloyd, J., \& Benedict, G. F. 2005b, in ASP Conf. Ser. 338, Astrometry in the Age of the Next Generation of Large Telescopes, ed. K. Seidelman \& A. Monet (San Francisco: ASP), 288

Readhead, A. C. S., Nakajima, T. S., Pearson, T. J., Neugebauer, G., Oke, J. B., \& Sargent, W. L. W. 1988, AJ, 95, 1278

Reid, I. N., Hawley, S. L., \& Gizis, J. E. 1995, AJ, 110, 1838

Salim, S., \& Gould, A. 2003, ApJ, 582, 1011

Troy, M., et al. 2000, Proc. SPIE, 4007, 31

Tuthill, P. G., Monnier, J. D., Danchi, W. C., Wishnow, E. H., \& Haniff, C. A. 2000, PASP, 112, 555

Weis, E. 1988, AJ, 96, 1710

Weistrop, D. 1981, AJ, 86, 1220

Wizinowich, P. L., et al. 2004, Proc. SPIE, 5490, 1 